BULL. AUSTRAL. MATH. SOC.

$18 \mathrm{~A} 40,18 \mathrm{~B} 30,18 \mathrm{C} 15$

VOL. 21 (1980), I69-173.

\title{
NOTE ON TOTAL CATEGORIES
}

\author{
WALTER THOLEN
}

It is shown that, for a semi-topological functor $T: A \rightarrow X$, the category $A$ is total, that is, the Yoneda embedding of $A$ has a left adjoint, if $X$ is total. In particular, monadic categories over Set (possibly without rank) are total, and full reflective subcategories of total categories are total.

\section{Total and compact categories}

A category A with small hom-classes is called total [6], if the Yoneda-embedding

$$
y_{\mathrm{A}}: \mathrm{A} \rightarrow \hat{\mathrm{A}}=\left[\mathrm{A}^{\mathrm{OP}}, \operatorname{Set}\right], A \mapsto \mathrm{A}(-, A),
$$

has a left adjoint. It is known [6] that any full reflective subcategory of a functor category [D, Set] with $D$ being small is total. In particular, monaic categories over Set with rank and their full reflective subcategories are total.

A total category $A$ is compact [3], that is, $A$ has small homclasses and any functor $U: A \rightarrow B$ preserving all existing colimits in $A$ has a right adjoint, provided $U$ is admissable [6] (that is, the homclasses $B(U A, B)$ are small for all $A \in \mathrm{Ob} A, B \in \mathrm{Ob} B)$. The reverse implication is false: it is proved in [2] that Adámek's [1] non-cocomplete (hence non-total) monadic category is compact.

Received 24 August 1979. The author thanks R.J. Wood whose talk on total categories at Oberwolfach 1979 drew his attention to this matter; the basic facts stated in Section 1 were learned there. Furthermore the author gratefully acknowledges stimulating discussion with $R$. Börger and R. Street. 
However, a cocompact category A (for example, any category satisfying the sufficient conditions of Freyd's Special Adjoint Functor Theorem) is total, provided A contains a generating set of objects. Namely, this last condition implies that $Y_{A}$ is co-admissible whence. ${ }_{A}$, preserving trivially all limits, has a left adjoint. Therefore, for categories which contain a generating set and a cogenerating set all notions total, cototal, compact, and cocompact coincide.

\section{The general lifting technique}

It is proved in [2] for a semi-topological [7] functor $T: A \rightarrow X$, that $A$ is compact if $X$ is. In the following we shall prove that a corresponding result holds for total categories. For this we consider a left adjoint $F: X \rightarrow A$ of $T$ and a natural equivalence $\varphi: \hat{F} \circ Y_{\mathrm{A}} \rightarrow Y_{X} \circ T$ (with $\hat{F}=\left[F^{\circ p}\right.$, Ens $\left.]\right):$

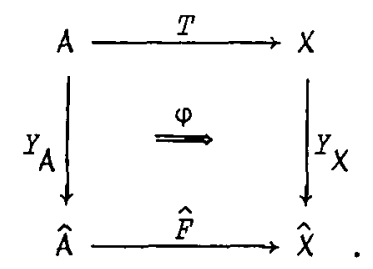

Let $Y_{X}$ have a left adjoint. According to the General Lifting Theorem 2.27 of [5], in order to prove right adjointness of $Y_{A}$ it suffices to prove that semi-initial factorizations of T-sources are locally respected by the above diagram. This means: if the commutative diagram (2),

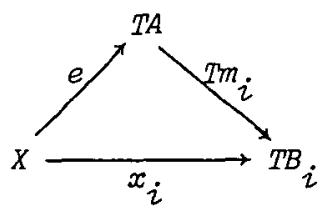

has the property that for any $z: T C \rightarrow X$ and all $b_{i}: C \rightarrow B_{i}$ with $x_{i} z=T b_{i}$ there is an $a: C \rightarrow A$ with $e z=T a$ and, therefore, $m_{i} a=b_{i}$ ("diagram (2) is T-semi-initial"), then diagram (3), 
(3)

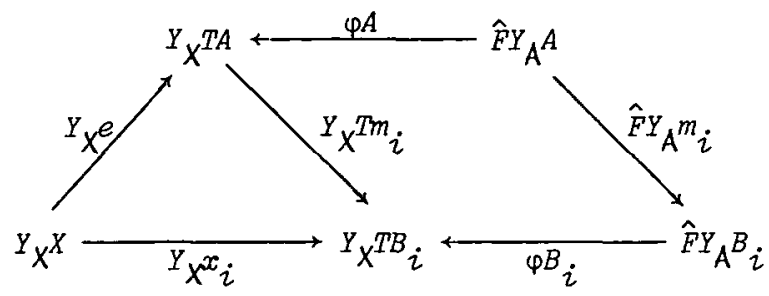

has the following property: for any $\zeta: \hat{F} H \rightarrow Y_{X} X \quad(H \in \mathrm{Ob} \hat{A})$ and all $B_{i}: H \rightarrow Y_{A} B_{i}$ with $\left(Y_{X} x_{i}\right) \zeta=\left(\varphi B_{i}\right)\left(\hat{F} B_{i}\right)$ there is an $\alpha: H \rightarrow Y_{A} A$ with $\left(Y_{X} \chi^{e} \zeta=(\varphi A)(\hat{F} \alpha)\right.$ and $\left(Y_{A} m_{i}\right) \alpha=B_{i} \quad$ ("diagram (3) is $\hat{F}$-semi-initial"). Usually $i$ ranges over a (proper) index class $I$. But the definition of semi-topological functors and the proof of 2.27 of [5] show that it does not matter, if $I$ belongs to any higher universe. In the present situation, one takes $I$ to be legitimate with respect to some universe for which $\hat{A}$ is legitimate.

\section{The lifting theorem}

THEOREM. Let $T: A \rightarrow X$ be a semi-topological functor. Then $A$ is total, if $X$ is total.

Proof. Let the T-semi-initial diagram (2) be given. In order to prove $\hat{F}$-semi-initiality of diagram (3) let $\zeta$ and $\beta_{i}$ be as above. For each $C \in O b A$ one then obtains the commutative diagram (4):

(4)

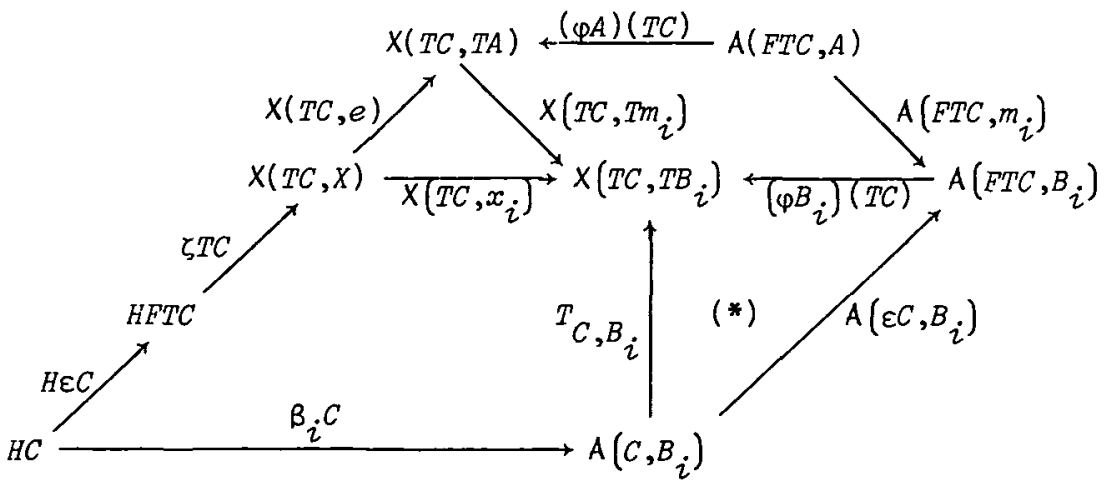

Here $\varepsilon$ denotes the co-unit of the adjoint pair $(F, T)$ such that the triangle (*) commutes for ${ }^{T}{ }_{C, B}$, being the respective restriction of $T$. 
For each $s \in H C$ one now has the commutative diagram (5):

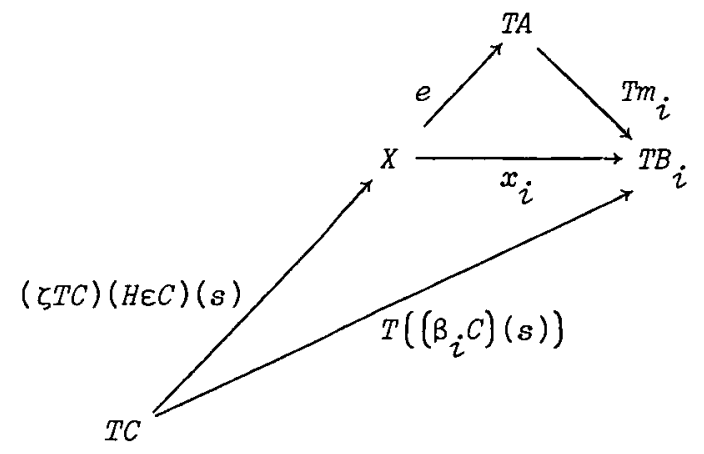

From the T-semi-initiality of (2) one therefore obtains a morphism $(\alpha C)(s): C \rightarrow A$ with $T((\alpha C)(s))=e((\zeta T C)(H \varepsilon C)(s))$. It is easily checked that, in this way, a natural transformation $\alpha: H \rightarrow Y_{A} A$ satisfying the needed equations is defined.

COROLLARY 1. Any monadic category over the category of sets is total.

COROLLARY 2. A fulz reflective subcategory of a total category is total.

\section{Final remark}

One is not able to prove a corresponding result as in the theorem for arbitrary monadic (instead of semi-topological) functors as Rattray [4] did in the case of compactness. To see this consider again the category of graphs over which Adámek [1] has constructed his non-total but monadic category: by the theorem, his base category, being semi-topological over sets, is total.

\section{References}

[1] Jirí Adámek, "Colimits of algebras revisited", BuZZ. Austral. Math. Soc. 17 (1977), 433-450.

[2] R. Börger, W. Tholen, M.B. Wischnewsky, H. Wolff, "Compactness and hypercompleteness", preprint. 
[3] John R. Isbell, "Small subcategories and completeness", Math. Systems Theory 2 (1968), 27-50.

[4] B.A. Rattray, "Adjoints to functors from categories of algebras", Comm. AZgebra 3 (1975), 563-569.

[5] R. Street, W. Tholen, M.B. Wischnewsky, H. Wolff, "Semitopological functors III: lifting of monads and adjoint functors", J. Pure Appl. Algebra (to appear).

[6] Ross Street and Robert Walters, "Yoneda structures on 2-categories", J. Algebra 50 (1978), 350-379.

[7] Walter Tholen, "Semi-topological functors I", J. Pure Appl. AZgebra 15 (1979), 53-73.

Fernuniversität,

Postfach 940,

D-5800 Hagen,

Federal Republic of Germany. 